\title{
A REPRESENTATION FOR THE SOLUTION OF FREDHOLM INTEGRAL EQUATIONS
}

H. H. KAGIWADA, R. E. KALABA AND A. SCHUMITZKY

Summary. This paper provides a new representation formula for the solution of Fredholm integral equations of the second kind. The formula has certain computational advantages over the usual representation involving the resolvent or the representation formula of Krein. As an application, the Bellman-Krein partial differential equation for the resolvent is derived.

Let $u$ be the unique solution of the Fredholm integral equation

$$
u(t, x)=g(t)+\int_{0}^{x} k(t, y) u(y, x) d y, \quad 0 \leqq t \leqq x ;
$$

it is assumed that $x$ is sufficiently small, $g$ and $k$ are continuous and

$$
k(t, y)=k(y, t) \text {. }
$$

The function $\Phi$ is the solution of the integral equation

$$
\Phi(t, x)=k(t, x)+\int_{0}^{x} k(t, y) \Phi(y, x) d y, \quad 0 \leqq t \leqq x,
$$

and the function $f$ is defined by the relation

$$
f(x)=\int_{0}^{x} \Phi(y, x) g(y) d y+g(x) .
$$

Then the solution of the integral Equation (1) may be represented in the form

$$
u(t, x)=f(t)+\int_{t}^{x} \Phi(t, w) f(w) d w, \quad 0 \leqq t \leqq x .
$$

The demonstration is simple. Differentiate both sides of Equation (1) with respect to $x$ to obtain

$$
u_{x}(t, x)=k(t, x) u(x, x)+\int_{0}^{x} k(t, y) u_{x}(y, x) d y .
$$

Equation (6) is regarded as an integral equation for the function $u_{x}$, having as its solution

Received by the editors September 19, 1968. 


$$
u_{x}(t, x)=\Phi(t, x) u(x, x) .
$$

Through integration this last equation becomes

$$
u(t, x)=u(t, t)+\int_{t}^{x} \Phi(t, w) u(w, w) d w .
$$

For $u(x, x)$ we may write

$$
u(x, x)=g(x)+\int_{0}^{x} k(x, y) u(y, x) d y .
$$

By cross-multiplication of Equations (1) and (3), integration on from $t=0$ to $t=x$ and use of the symmetry of the kernel $k$, it is seen that

$$
\int_{0}^{x} \Phi(t, x) g(t) d t=\int_{0}^{x} u(t, x) k(t, x) d t .
$$

Equation (9) becomes

$$
u(x, x)=g(x)+\int_{0}^{x} \Phi(t, x) g(t) d t, \quad 0 \leqq x .
$$

Equation (8) then assumes the desired form

$$
\begin{aligned}
u(t, x)= & g(t)+\int_{0}^{t} \Phi\left(t^{\prime}, t\right) g\left(t^{\prime}\right) d t^{\prime} \\
& +\int_{\imath}^{x} \Phi(t, w)\left[g(w)+\int_{0}^{w} \Phi\left(t^{\prime}, w\right) g\left(t^{\prime}\right) d t^{\prime}\right] d w .
\end{aligned}
$$

The above formulas provide the solution of the integral Equation (1) in terms of the function $\Phi$ and two integrations. The function $\Phi$ is a function of two variables, and $u$ is given for all $0 \leqq t \leqq x<c$, where $c$ is the first critical length for the kernel $k$.

The resolvent $K$ yields the solution of Equation (1) in the form

$$
u(t, x)=g(t)+\int_{0}^{x} K(t, y, x) g(y) d y, \quad 0 \leqq t \leqq x .
$$

The function $K$ is known to be symmetric

$$
K(t, y, x)=K(y, t, x),
$$

and satisfies the integral Equation (1) 


$$
K(t, y, x)=k(t, y)+\int_{0}^{x} k\left(t, y^{\prime}\right) K\left(y^{\prime}, y, x\right) d y^{\prime} .
$$

Comparing Equation (15) with the earlier Equation (3) for $\Phi$, it is seen that

$$
\Phi(t, x)=K(t, x, x)=K(x, t, x) .
$$

Notice, however, that $K$ is a function of three variables. By suppressing $x, K$ may be regarded as a function of two variables. It yields $u$ as a function of $t$ for this fixed $x$, whereas $\Phi$ yields $u$ for all $x \leqq c$.

As an application of our representation formula, let

$$
g(t)=k\left(t, y^{\prime}\right)
$$

in Equation (1). It is known that the solution $u$ is the resolvent

$$
u(t, x)=K\left(t, y^{\prime}, x\right) .
$$

Use of the representation formula, Equation (12), shows that

$$
K(t, y, x)=\Phi(y, t)+\int_{t}^{x} \Phi(t, w) \Phi(y, w) d w, \quad 0 \leqq y \leqq t \leqq x .
$$

Differentiation with respect to $x$ then provides the Bellman-Krein formula [2], [3]

$$
K_{x}(t, y, x)=\Phi(t, x) \Phi(y, x),
$$

or

$$
K_{x}(t, y, x)=K(t, x, x) K(x, y, x) .
$$

Krein [3] provides a representation formula for the solution of Equation (1). His approach consists in letting $s$ be the solution corresponding to the inhomogeneous term $g(t) \equiv 1$. If $s(x, x) \neq 0$, then

$$
\Phi(t, x)=s_{x}(t, x) / s(x, x) .
$$

In addition it is observed that

$$
\begin{aligned}
\frac{d}{d x} \int_{0}^{x} s(t, x) g(t) d t & =s(x, x) g(x)+\int_{0}^{x} s_{x}(t, x) g(t) d t \\
& =s(x, x)\left[g(x)+\int_{0}^{x} \Phi(t, x) g(t) d t\right] \\
& =s(x, x) u(x, x) .
\end{aligned}
$$


In the above equation use has been made of Equation (16). Returning to Equation (8), Krein's formula is obtained:

$$
\begin{aligned}
u(t, x)= & \frac{\frac{d}{d t} \int_{0}^{t} s\left(t^{\prime}, t\right) g\left(t^{\prime}\right) d t^{\prime}}{s(t, t)} \\
& +\int_{t}^{x} \frac{s_{w}(t, w)}{s(w, w)} \frac{\frac{d}{d w} \int_{0}^{w} s\left(t^{\prime}, w\right) g\left(t^{\prime}\right) d t^{\prime}}{s(w, w)} d w .
\end{aligned}
$$

This representation contains indicated differentiations as well as integrations, a drawback from the computational viewpoint.

The importance of the function $\Phi$ in radiative transfer is stressed in references [4]-[10]. There it is also shown that efficient methods exist for determining this basic function in a variety of cases; see also [11].

\section{REFERENCES}

1. R. Courant and D. Hilbert, Methods of mathematical physics, Vol. 1, Interscience, New York, 1953.

2. R. Bellman, Functional equations in the theory of dynamic programming-VII: $A$ partial differential equation for the Fredholm resolvent, Proc. Amer. Math. Soc. 8 (1957), 435-440.

3. M. G. Krein, On a new method for solving linear integral equations of the first and second kinds, Dokl. Akad. Nauk SSSR 100 (1955), 413-416.

4. H. Kagiwada and R. Kalaba, An initial-value method for Fredholm integral equations of convolution type, The RAND Corporation, RM-5186-PR, 1966.

5. H. Kagiwada, R. Kalaba and S. Ueno, Evaluation of functionals of solutions of Fredholm integral equations with displacement kernels, The RAND Corporation, RM5459-PR, 1967. 1963.

6. V. V. Sobolev, A treatise on radiative transfer, Van Nostrand, Princeton, N. J.,

7. H. Kagiwada and R. Kalaba, A new initial-value method for internal intensities in radiative transfer, Astrophys. J. 147 (1967), 301-309.

8. S. Ueno, The invariant imbedding method for transport problems-II. Resolvent in photon diffusion equation, J. Math. Anal. Appl. 3 (1961), 361-372.

9. H. Kagiwada and R. Kalaba, An initial-value method suitable for the computation of certain Fredholm resolvents, J. Math. and Phys. Sci. (1967), 109-122.

10. H. Kagiwada, R. Kalaba and A. Schumitzky, An initial-value method for general Fredholm integral equations, J. Math. Anal. Appl. 19 (1967), 197-203.

11. A. Schumitzky, On the equivalence between matrix Riccati equations and Fredholm resolvents, J. Comput. System Sci. 2 (1968), 76-87.

The Rand Corporation, Santa Monica and

University of Southern California, los Angeles 